\title{
Stark broadening data for spectral lines of rare-earth elements: Example of Tb II and Tb IV
}

\author{
Milan S. Dimitrijević ${ }^{1,2}$ \\ 1 Astronomical Observatory, Volgina 7, 11060 Belgrade 38, Serbia \\ 2 Sorbonne Université, Observatoire de Paris, Université PSL, CNRS, \\ LERMA, F-92190 Meudon, France
}

Received: August 14, 2019; Accepted: September 28, 2019

\begin{abstract}
Stark full widths at half maximum (FWHM) for five multiplets of $\mathrm{Tb}$ II and 8 multiplets of Tb IV have been calculated for electron density of $10^{17} \mathrm{~cm}^{-3}$ by using the symplified modified semiempirical (SMSE) method. The calculations were performed for temperatures from $5000 \mathrm{~K}$ to $80000 \mathrm{~K}$, in the region where the used theory is applicable.
\end{abstract}

Key words: Spectral lines - Plasma - Atomic data - Stark broadening

\section{Introduction}

The lanthanides are a series of 15 chemical elements with atomic numbers 57 through 71 , from lanthanum to lutetium. Along with the chemically similar elements scandium and yttrium, they are often collectively known as the rare earth elements (REE). Stark broadening of rare-earth atom and ion spectral lines is of considerable importance in astrophysics due to rare-earth peak of abundance distribution of chemical elements. Consequently, a lot of spectral lines of these elements have been and will be observed in stellar spectra, in particular due to the development of space astronomy. For example, instruments like the Goddard High Resolution Spectrograph (GHRS) on the Hubble Space Telescope, enable us to obtain spectral lines with unprecedented resolution and accuracy.

The data on Stark broadening of rare-earth elements are scarce, first because of the lack of reliable atomic data for the corresponding calculations. The semiclassical perturbation theory (SCP) for Stark broadening of isolated lines (Sahal-Bréchot, 1969a,b) can be used only for some scandium and yttrium ions. In some cases the modified semiempirical method (MSE - Dimitrijević \& Konjević 1980) or the symplified modified semiempirical method (SMSA - Dimitrijević \& Konjević 1987) can be applied, but in many cases there is not enough atomic data even for the simplest calculations and regularities and systematic trends can only be used for very rough estimates (Popović \& Dimitrijević, 1998). Concerning Stark broadening data for REE spectral lines, up to now data are 
published for six multiplets of Sc II, calculated by using MSE (Popović \& Dimitrijević, 1996), ten of Sc III, using SCP (Dimitrijević \& Sahal-Bréchot, 1992), four of Sc X and ten of Sc XI, using SCP (Dimitrijević \& Sahal-Bréchot, 1998a), six of Y II using MSE (Popović \& Dimitrijević, 1996), 32 of Y III, using SCP (Dimitrijević \& Sahal-Bréchot, 1998b), three of La II, six of La III, 284 of Nd II, by using SMSE (Popović et al., 2001b) seven of Eu II, by using MSE, one of Eu III by using SMSE (Popović et al., 1999), four of Yb III, by using MSE (Dimitrijević, 2019), 27 of Lu III, by using MSE (Majlinger et al., 2015) and four of Lu IV, by using MSE, (Dimitrijević, 2019)

The significance of Strak broadening data, including data for REE elemts, is increasing in astrophysics due to the increasing possibility of obtaining high resolution spectra. Rauch et al. (2007) underlined that they "are of crucial importance for sophisticated analysis of stellar spectra by means of NLTE model atmospheres". Spectral lines of neutral and ionized terbium are present and observed in stellar spectra. For example, the terbium abundance was derived by Siqueira Mello et al. (2014) for the moderately r-process-enhanced star CS 30315-029, based on three weak Tb II lines. Elkin et al. (2015) found Tb III lines in ro Ap star HD 213637 and Sachkov et al. (2008) in ro Ap star 10 Aql. $\mathrm{Tb}$ I, Tb II and Tb III lines have been found and in the spectrum of the extreme roAp star HD 101065, known as Przybylski's star (Cowley et al., 2000).

Since there is neither experimental or theoretical data for Stark broadening of $\mathrm{Tb}$ II and Tb IV, we calculated here Stark full widths at half maximum (FWHM) for five transitions of Tb II and eight transitions of Tb IV by using the simplified modified semiempirical method (Dimitrijević \& Konjević, 1987), since there is not enough atomic data for more advanced calculations.

\section{Notes about calculations}

In cases when we do not have enough data for more sophisticated theoretical calculations, or when, for example in astrophysics for model atmosphere or radiative transfer calculations, a lot of spectral lines, with the corresponding data should be taken into account. Here the simplified modified semiempirical formula (Dimitrijević \& Konjević, 1987) for Stark widths of isolated, singly, and multiply charged ion lines might be very useful. This formula may be used in the case when the nearest atomic energy level, having an allowed dipole transition from, or to, the initial or final energy level of the spectral line considered is far enough away so that the influence of elastic collisions is dominant.

By using the symplified modified semiempirical (SMSE) method (Dimitrijević \& Konjević, 1987), the most advanced method applicable in present case due to the lack of a corresponding set of atomic data, we calculated Stark full widths at half maximum (FWHM) due to collisions with electrons, for five transitions of Tb II and eight of Tb IV, for an electron density of $10^{17} \mathrm{~cm}^{-3}$ and for a temperature interval from $5000 \mathrm{~K}$ up to $20000 \mathrm{~K}$ or $40000 \mathrm{~K}$, depending 
on the limits of validity of the SMSE method, in the case of Tb II, while for $\mathrm{Tb}$ IV the temperature interval is from $5000 \mathrm{~K}$ to $80000 \mathrm{~K}$. Energy levels and ionization potentials for these calculations have been taken from Martin et al. (1978) and Kramida et al. (2019).

\section{Results and discussion}

Table 1. This table gives electron-impact broadening (Stark broadening) Full Widths at Half Intensity Maximum (W) for Tb II spectral lines, for a perturber density of $10^{17} \mathrm{~cm}^{-3}$ and temperatures from 5000 to $40000 \mathrm{~K}$. Also is given quantity $3 k T / 2 \Delta E$, where $\Delta E$ is the energy difference between closest perturbing level and the closer of initial and final levels.

\begin{tabular}{|c|c|c|c|}
\hline Transition & $\overline{\text { Temp. }}$ & $\bar{W} \mathrm{~W}[\AA]$ & $3 \mathrm{k \textrm {kT }} / 2 \Delta \mathrm{E}$ \\
\hline \multirow{3}{*}{$\begin{array}{c}\text { Tb II }\left({ }^{6} \mathrm{H}_{15 / 2}^{o}\right) 6 \mathrm{~s}_{1 / 2}(15 / 2,1 / 2)- \\
\left({ }^{6} \mathrm{H}_{15 / 2}^{o}\right) 6 \mathrm{p}_{1 / 2}(15 / 2,1 / 2) \\
3934.1\end{array}$} & 5000. & 0.393 & 0.734 \\
\hline & 10000. & 0.278 & 1.47 \\
\hline & 20000. & 0.196 & 2.94 \\
\hline \multirow{3}{*}{$\begin{array}{c}\mathrm{Tb} \text { II }\left({ }^{6} \mathrm{H}_{15 / 2}^{o}\right) 6 \mathrm{~s}_{1 / 2}(15 / 2,1 / 2)- \\
\left({ }^{6} \mathrm{H}_{15 / 2}^{o}\right) 6 \mathrm{p}_{3 / 2}(15 / 2,3 / 2) \\
3610.5\end{array}$} & 5000 . & 0.350 & 0.556 \\
\hline & 10000 . & 0.247 & 1.11 \\
\hline & 20000 . & 0.175 & 2.22 \\
\hline \multirow{3}{*}{$\begin{array}{c}\text { Tb II }\left({ }^{6} \mathrm{H}_{13 / 2}^{o}\right) 6 \mathrm{~s}_{1 / 2}(13 / 2,1 / 2)- \\
\left({ }^{6} \mathrm{H}_{13 / 2}^{o}\right) 6 \mathrm{p}_{1 / 2}(13 / 2,1 / 2) \\
3938.3\end{array}$} & 5000 . & 0.393 & 0.529 \\
\hline & 10000 . & 0.278 & 1.06 \\
\hline & 20000 . & 0.196 & 2.12 \\
\hline \multirow{4}{*}{$\begin{array}{c}\mathrm{Tb} \text { II }\left({ }^{6} \mathrm{H}_{13 / 2}^{o}\right) 6 \mathrm{~s}_{1 / 2}(13 / 2,1 / 2)- \\
\left({ }^{6} \mathrm{H}_{13 / 2}^{o}\right) 6 \mathrm{p}_{3 / 2}(13 / 2,3 / 2) \\
3567.3\end{array}$} & 5000. & 0.344 & 0.417 \\
\hline & 10000. & 0.243 & 0.834 \\
\hline & 20000 . & 0.172 & 1.67 \\
\hline & 40000 . & 0.122 & 3.34 \\
\hline \multirow{4}{*}{$\begin{array}{c}\mathrm{Tb} \text { II }\left({ }^{6} \mathrm{H}_{11 / 2}^{o}\right) 6 \mathrm{~s}_{1 / 2}(11 / 2,1 / 2)- \\
\left({ }^{6} \mathrm{H}_{11 / 2}^{o}\right) 6 \mathrm{p}_{1 / 2}(11 / 2,1 / 2) \\
3966.1\end{array}$} & 5000. & 0.397 & 0.449 \\
\hline & 10000 . & 0.281 & 0.897 \\
\hline & 20000 . & 0.198 & 1.79 \\
\hline & 40000 . & 0.140 & 3.59 \\
\hline
\end{tabular}

The results obtained for Stark widths for Tb II spectral lines are presented in Table 1 and those for Tb IV are in Table 2. The wavelengths given in Tables 1 and 2 are calculated from the energy levels used. In the last column is $3 k T / 2 \Delta E$, representing the ratio of the average energy of free electrons, $E=3 k T / 2$, and the energy difference between the initial $(i)$ or final $(f)$ and the closest perturbing 
Table 2. Same as in Table 1, but for Tb IV, for temperature interval 5000 - $80000 \mathrm{~K}$.

\begin{tabular}{|c|c|c|c|}
\hline Transition & Temp. & $\mathrm{W}[\AA]$ & $3 \mathrm{kT} / 2 \Delta \mathrm{E}$ \\
\hline Tb IV $\left({ }^{8} \mathrm{~S}^{o}\right) 5 \mathrm{~d}{ }^{9} \mathrm{D}^{o}-$ & 5000. & $0.275 \mathrm{E}-01$ & 0.122 \\
\hline \multirow{4}{*}{$\begin{array}{c}\left({ }^{8} \mathrm{~S}_{7 / 2}^{o}\right) 6 \mathrm{p}_{1 / 2}(7 / 2,1 / 2)_{3} \\
1338.7\end{array}$} & 10000. & $0.195 \mathrm{E}-01$ & 0.243 \\
\hline & 20000. & $0.138 \mathrm{E}-01$ & 0.486 \\
\hline & 40000. & $0.974 \mathrm{E}-02$ & 0.973 \\
\hline & 80000. & $0.689 \mathrm{E}-02$ & 1.95 \\
\hline \multirow{5}{*}{$\begin{array}{c}\mathrm{Tb} \operatorname{IV}\left({ }^{8} \mathrm{~S}^{o}\right) 5 \mathrm{~d}^{9} \mathrm{D}^{o}- \\
\left({ }^{8} \mathrm{~S}_{7 / 2}^{o}\right) 6 \mathrm{p}_{1 / 2}(7 / 2,1 / 2) 4 \\
1324.5\end{array}$} & 5000. & $0.273 \mathrm{E}-01$ & 0.119 \\
\hline & 10000. & $0.193 \mathrm{E}-01$ & 0.239 \\
\hline & 20000 . & $0.136 \mathrm{E}-01$ & 0.477 \\
\hline & 40000. & $0.964 \mathrm{E}-02$ & 0.955 \\
\hline & 80000 . & $0.682 \mathrm{E}-02$ & 1.91 \\
\hline \multirow{5}{*}{$\begin{array}{c}\mathrm{Tb} \text { IV }\left({ }^{8} \mathrm{~S}^{o}\right) 5 \mathrm{~d}{ }^{9} \mathrm{D}^{o}- \\
\left({ }^{8} \mathrm{~S}_{7 / 2}^{o}\right) 6 \mathrm{p}_{1 / 2}(7 / 2,3 / 2)_{5} \\
1323.3\end{array}$} & 5000 & $0.255 \mathrm{E}-01$ & 0.106 \\
\hline & 10000. & $0.180 \mathrm{E}-01$ & 0.211 \\
\hline & 20000. & $0.128 \mathrm{E}-01$ & 0.423 \\
\hline & 40000. & $0.902 \mathrm{E}-02$ & 0.845 \\
\hline & 80000. & $0.638 \mathrm{E}-02$ & 1.69 \\
\hline \multirow{5}{*}{$\begin{array}{c}\mathrm{Tb} \text { IV }\left({ }^{8} \mathrm{~S}^{o}\right) 6 \mathrm{~s}{ }^{9} \mathrm{~S}^{o}- \\
\left({ }^{8} \mathrm{~S}_{7 / 2}^{o}\right) 6 \mathrm{p}_{1 / 2}(7 / 2,1 / 2)_{3} \\
2331.8\end{array}$} & 5000. & 0.161 & 0.122 \\
\hline & 10000. & 0.114 & 0.243 \\
\hline & 20000. & $0.807 \mathrm{E}-01$ & 0.486 \\
\hline & 40000. & $0.570 \mathrm{E}-01$ & 0.973 \\
\hline & 80000. & $0.403 \mathrm{E}-01$ & 1.95 \\
\hline \multirow{5}{*}{$\begin{array}{c}\mathrm{Tb} \text { IV }\left({ }^{8} \mathrm{~S}^{o}\right) 6 \mathrm{~s}^{9} \mathrm{~S}^{o}- \\
\left({ }^{8} \mathrm{~S}_{7 / 2}^{o}\right) 6 \mathrm{p}_{1 / 2}(7 / 2,1 / 2)_{4} \\
2289.3\end{array}$} & 5000 & 0.156 & 0.122 \\
\hline & 10000. & 0.111 & 0.243 \\
\hline & 20000. & $0.782 \mathrm{E}-01$ & 0.486 \\
\hline & 40000 . & $0.553 \mathrm{E}-01$ & 0.973 \\
\hline & 80000 . & $0.391 \mathrm{E}-01$ & 1.95 \\
\hline \multirow{5}{*}{$\begin{array}{c}\mathrm{Tb} \operatorname{IV}\left({ }^{8} \mathrm{~S}^{o}\right) 6 \mathrm{~s}{ }^{9} \mathrm{~S}^{o}- \\
\left({ }^{8} \mathrm{~S}_{7 / 2}^{o}\right) 6 \mathrm{p}_{3 / 2}(7 / 2,3 / 2)_{5} \\
2027.1\end{array}$} & 5000. & 0.128 & 0.122 \\
\hline & 10000. & $0.904 \mathrm{E}-01$ & 0.243 \\
\hline & 20000. & $0.639 \mathrm{E}-01$ & 0.486 \\
\hline & 40000 . & $0.452 \mathrm{E}-01$ & 0.973 \\
\hline & 80000. & $0.319 \mathrm{E}-01$ & 1.95 \\
\hline \multirow{5}{*}{$\begin{array}{c}\mathrm{Tb} \text { IV }\left({ }^{8} \mathrm{~S}^{o}\right) 6 \mathrm{~s}{ }^{9} \mathrm{~S}^{o}- \\
\left({ }^{8} \mathrm{~S}_{7 / 2}^{o}\right) 6 \mathrm{p}_{3 / 2}(7 / 2,3 / 2) \\
1203.8\end{array}$} & 5000. & $0.250 \mathrm{E}-01$ & 0.107 \\
\hline & 10000 . & $0.177 \mathrm{E}-01$ & 0.214 \\
\hline & 20000. & $0.125 \mathrm{E}-01$ & 0.429 \\
\hline & 40000. & $0.884 \mathrm{E}-02$ & 0.858 \\
\hline & 80000 . & $0.625 \mathrm{E}-02$ & 1.72 \\
\hline \multirow{5}{*}{$\begin{array}{c}\mathrm{Tb} \text { IV }\left({ }^{8} \mathrm{~S}^{o}\right) 5 \mathrm{~d}{ }^{7} \mathrm{D}^{o}- \\
\left({ }^{8} \mathrm{~S}_{7 / 2}^{o}\right) 6 \mathrm{p}_{3 / 2}(7 / 2,3 / 2 \\
2056.2\end{array}$} & 5000. & 0.135 & 0.107 \\
\hline & 10000. & $0.953 \mathrm{E}-01$ & 0.214 \\
\hline & 20000. & $0.674 \mathrm{E}-01$ & 0.429 \\
\hline & & $0.477 \mathrm{E}-01$ & 0.858 \\
\hline & 80000. & $0.337 \mathrm{E}-01$ & 1.72 \\
\hline
\end{tabular}


level $\left(i^{\prime}\right.$ or $\left.f^{\prime}\right)$. Here, $T$ is the temperature and $k$ Boltzmann constant. The larger of these values for the initial and final levels are taken, i.e.:

$$
\Delta E=\operatorname{Max}\left[E / \Delta E_{i, i^{\prime}}, E / \Delta E_{f, f^{\prime}}\right]
$$

This is the validity condition for the SMSE method used. The $3 k T / 2 \Delta E$ is equal to one at the threshold for the corresponding inelastic transition. If it is lower, than elastic collisions are dominant and the SMSE method is completely applicable and valid. For values larger than one, the inelastic collisions start become more and more important with its increase. Up to the value of around two, the results are acceptable. Data for the slightly higher values in Table 1, are given in order to enable better interpolation. The behavior of Starks widths with electron density is linear within the limits of validity of the SMSE method. These are the first published data for Stark width of Tb II and Tb IV and there is no other theoretical or experimental data to compare with.

\section{Conclusion}

New Stark FWHM for five lines of singly charged, and eight lines for triply charged terbium ion have been calculated in this work using the simplified modified semiempirical formula (Dimitrijević \& Konjević, 1987). There is no other theoretical or experimental Stark broadening data for these emitters, so that the data presented are of interest for stellar plasma analysis, as well as for laboratory plasma diagnostics and laser produced plasma investigation.

\section{References}

Cowley, C. R., Ryabchikova, T., Kupka, F., Bord, D. J., Mathys, G., \& Bidelman, W. P. 2000, Abundances in Przybylski's star. MNRAS, 317, 299309.

Dimitrijević, M. S. 2019, Stark widths of Yb III and Lu IV spectral lines, Atoms, $7,10$.

Dimitrijević, M. S., \& Konjević, N., Stark widths of doubly- and triply-ionized atom lines. 1980, J. Quant. Spectrosc. Radiat. Transf., 24, 451.

Dimitrijević, M.S., \& Konjević, N. 1987. Simple estimates for Stark broadening of ion lines in stellar plasma. A\&A, 172, 345-349.

Dimitrijević, M. S., \& Sahal-Bréchot, S. 1992. Stark Broadening of Spectral Lines of Multicharged Ions of Astrophysical Interest. IV. Sc III and Ti IV Lines. A\&AS, 95, 121-128.

Dimitrijević, M. S., \& Sahal-Bréchot, S. 1998a. Stark broadening of spectral lines of multicharged ions of astrophysical interest. XXI. Sc X, Sc XI, Ti XI and Ti XII spectral lines. A\&AS, 131, 143-144.

Dimitrijević, M. S., \& Sahal-Bréchot, S. 1998b. Stark Broadening of Y III Spectral Lines. J. Appl. Spectrosc., 65, 492-498. 
Elkin, V. G., Kurtz, D. W., \& Mathys, G. 2015, Time resolved spectroscopy of the cool Ap star HD 213637. MNRAS, 446, 41264131.

Kramida, A., Ralchenko, Yu., Reader, J.,\& - NIST ASD Team 2018, NIST Atomic Spectra Database. Gaithersburg, MD: National Institute of Standards and Technology. (ver. 5.6.1), [Online]. Available: https://physics.nist.gov/asd [2019, August 13].

Majlinger, Z., Simić, Z., \& Dimitrijević, M. S. 2015, On the Stark Broadening of Lu III Spectral Lines. J. Astrophys. Astron., 36, 671.

Martin, W. C., Zalubas, R., \& Hagan, L. 1978, Atomic Energy Levels The Rare-Earth Elements, Nat. Stand. Ref. Data Ser., NSRDS-NBS 60, 1-422.

Popović, L. Č., \& Dimitrijević, M. S. 1996, Stark widths for astrophysically important ns-np transitions in Sc II, Y II and Zr II spectra. A\&AS, 120, 373-374.

Popović, L. Č., \& Dimitrijević, M. S. 1998, A program for electron-impact broadening parameter calculations of ionized rare-earth element lines. Contrib. Astron. Obs. Skalnaté Pleso, 27, 353-355.

Popović, L. Č., Dimitrijević, M.S., \& Ryabchikova, T. 1999, The electron-impact broadening effect in CP stars: The case of La II, La III, Eu II, and Eu III lines. A\&A, 350, 719724.

Popović, L. Č., Simić, S., Milovanović, N., \& Dimitrijević, M. S. 2001, Stark Broadening Effect in Stellar Atmospheres: Nd II Lines. 2001, ApJS, 135, 109.

Rauch, T., Ziegler, M., Werner, K., Kruk, J. W., Oliveira, C. M., Putte, D. V., Mignani, R. P., \& Kerber, F. 2007, High-resolution FUSE and HST ultraviolet spectroscopy of the white dwarf central star of Sh 2-216. Astrophysics, 470, 317329 .

Sachkov, M., Kochukov, O., Ryabchikova, T., Leone, F., Bagnulo, S., \& Weiss, W. W. 2008. Spectroscopic study of pulsations in the atmosphere of roAp star 10 Aql. Contrib. Astron. Obs. Skalnaté Pleso, 38, 323-328.

Sahal-Bréchot, S. 1969a, Impact Theory of the Broadening and Shift of Spectral Lines due to Electrons and Ions in a Plasma. A\&A, 1, 91-123.

Sahal-Bréchot, S. 1969b, Impact Theory of the Broadening and Shift of Spectral Lines due to Electrons and Ions in a Plasma (Continued). A\&A, 2, 322-354.

Simić, Z., Dimitrijević, M. S., \& Popović, L. Č. 2014, Stark broadening data for spectral lines of rare-earth elements: Nb III. Adv. Space Res., 54, 1231.

Siqueira Mello, C., Hill, V., Barbuy, B., Spite, M., Spite, F., Beers, T. C., Caffau, E., Bonifacio, P., Cayrel, R., François, P., Schatz, H., \& Wanajo, S. 2014, High-resolution abundance analysis of very metal-poor r-I stars. A\&A, 565, A93. 